\section{Reprogramming of early embryonic blastomeres into endodermal progenitors by a Caenorhabditis elegans GATA factor}

\author{
Jiangwen Zhu, ${ }^{1}$ Tetsunari Fukushige, ${ }^{2}$ \\ James D. McGhee, ${ }^{2}$ and Joel H. Rothman ${ }^{1,3}$ \\ ${ }^{1}$ Department of Molecular, Cellular, and Developmental \\ Biology and Neuroscience Research Institute, University of \\ California, Santa Barbara, California 93106 USA; ${ }^{2}$ Department \\ of Biochemistry and Molecular Biology, University of Calgary, \\ Calgary, Alberta T2N 4N1, Canada
}

The END-1 GATA factor has been implicated in specifying endoderm in Caenorhabditis elegans and is the earliest known zygotic protein expressed in the lineage of $E$, the clonal endoderm progenitor. We report that ubiquitous end-1 expression during a critical period in embryogenesis causes all non-endodermal lineages to produce endoderm instead of ectoderm and/or mesoderm. END-1 expression bypasses the requirement for maternal SKN-1 and the maternal Wnt signaling pathway in endoderm formation. This suggests that a primary function of these maternal factors is to regulate zygotic end-1 expression, which is then sufficient to initiate the entire program for endoderm development.

Received September 2, 1998; revised version accepted October 19, 1998.

How and when during development is a cell committed to a particular fate? Studies on embryos of the nematode Caenorhabditis elegans reveal that blastomeres appear to become committed to distinct patterns of differentiation during the first few embryonic cleavages by the combined action of cell-cell interactions and cell-autonomous maternal factors (for review, see Schnabel and Priess 1997; Bowerman 1998). These events control the identity of the embryonic founder cells, each of which gives rise to a unique set of differentiated cell types. The ability to contribute to each germ layer is differentially allocated to the founder cells. For example, production of endoderm, consisting of only the intestine, is confined to a single founder cell, the E blastomere, in the seven-cell embryo (Sulston et al. 1983). The E cell identity is determined by maternally contributed factors, including the SKN-1 transcription factor (Bowerman et al. 1992, 1993; Blackwell et al. 1994) and the Wnt signaling pathway, which causes the E cell parent, EMS, to undergo an asymmetric division that segregates endoderm and me-

[Key Words: C. elegans; endoderm; end-1; GATA factor; germ layer; cell fate]

${ }^{3}$ Corresponding author.

E-MAIL rothman@lifesci.ucsb.edu; FAX (805) 893-2005. soderm lineages (Rocheleau et al. 1997; Thorpe et al. 1997). When either maternal SKN-1 activity or the maternal Wnt signal transduction pathway is eliminated, the E cell adopts the fate of another early blastomere and endoderm development does not occur.

Specification of endoderm in C. elegans also requires zygotic expression of a genomic region identified by chromosomal deletions (Zhu et al. 1997). Although there appear to be multiple genes in this region that regulate endoderm development (Zhu et al. 1997; E. NewmanSmith, M. Maduro, and J. Rothman, unpubl.), the end-1 gene can restore endoderm production to embryos carrying deletions of the region. end-1 is normally expressed specifically in the E cell and early E lineage. end-1 encodes an apparent zinc finger transcription factor of the GATA factor family (Zhu et al. 1997). GATA factors have also been implicated in regulating endoderm development in Drosophila (Reuter 1994; Rehorn et al. 1996), and in the developing endoderm of vertebrates (Laverriere et al. 1994; Soudais et al. 1995). Moreover, at least two other GATA factors, in addition to END-1, function in endoderm differentiation in C. elegans (Fukushige et al. 1998; M. Maduro, E. Newman-Smith, and J. Rothman, unpubl.). These findings suggest that endoderm is established by a conserved mechanism in all triploblastic animals.

The maternal factors required for endoderm development may participate directly in specifying the identity of the E cell, or they might simply function to promote expression of zygotic cell fate regulators in the E lineage, for example by imparting the highly restricted E-lineagespecific expression to end-1. In the latter case, end-1 may be capable of directing endoderm development irrespective of the ancestry of a cell or the activity of these maternal factors.

Here, we ask whether expression of a single zygotic factor, END-1, is sufficient to redirect the developmental fate of early blastomeres. We report that expression of END-1 in nonendodermal lineages of early C. elegans embryos results in their respecification into endodermal lineages. We find that embryonic blastomeres are competent to undergo endoderm development several cell divisions after the stage at which their lineages are normally committed to an exclusively nonendodermal lineage. Expression of end-1 under control of a heat shock promoter bypasses the requirement for the maternal endoderm-determining factors, suggesting that a primary requirement for maternal regulators of endoderm development is to activate end-1 and restrict its expression.

\section{Results and Discussion}

END-1 can promote widespread endoderm differentiation

The end-1 coding region was removed from its normal transcriptional regulatory sequences and expressed under heat shock control (see Materials and Methods). Embryos carrying these constructs were given a heat shock 
Zhu et al.

Figure 1. Widespread intestinal differentiation in embryos expressing end-1 ectopically. $(A, C, E)$ Birefringent gut granules under dark-field polarized light. $(B, D, F)$ Overall morphology seen by Nomarski optics. (Arrowhead) Gut nuclei; (arrow) brush border. $(B)$ The granules in the embryos shown in $C$ were much fainter than those in $A$ and $E$, in which gut is localized to one region $(B, F)$. A massive flux of yolk material into the developing endoderm occurs during normal embryogenesis, coincident with production of birefringent granules (Bossinger and Schierenberg 1992); in embryos containing mostly endoderm cells, this concentration of material cannot occur, probably accounting for the overall faintness of granules in such embryos. [The ectopic brush border structures $(D)$ are readily apparent when viewed in three dimensions while observing embryos at the microscope but are not as clearly discerned in a single focal plane, as shown here and in Fig. 5.] When hs-end-1 embryos were heat-shocked at a later time ( 86- to 170-cell stage), bright granules were limited to the region of the normal gut $(E)$. The morphology of a representative embryo (arrow in $E$ ) is shown in $F$; the cells in the region lacking gut granules are not gut-like (cf. $D$ ). Heat-shocked embryos carrying a control hs-Ce-dad-1 construct or hs-elt-1 never showed ectopic gut granules (Sugimoto et al. 1995; Fukushige et al. 1998; data not shown). (G-L) Immunofluorescence analyses of partially elongated wild-type $(G)$, elongated wild-type $(I, K)$, and terminal heat-shocked hs-end-1 embryos $(H, J, L)$. Anti-ELT-2 antibody $(G, H)$; monoclonal antibody 1CB4 $(I, J)$, and mAb MH33 (Waterson 1998) $K, L) .(M, N)$ Embryos histochemically stained for GES-1 esterase activity (Edgar and McGhee 1986) show expression of the enzyme restricted to the gut in wild-type embryos $(M)$ but throughout heat-shocked hs-end-1 embryos (N). Bar in $A, \sim 10 \mu \mathrm{m}$; bar in $E$, $(C, E) \sim 50 \mu \mathrm{m}$.

to activate expression of end-1 throughout the embryo (Fig. 1). Following recovery from the heat shock, cells throughout the differentiated hs-end-1 embryos were found to contain birefringent granules, characteristic of gut cells (Siddiqui et al. 1980) (Fig. 1, cf. C with A and E), as well as structures resembling intestinal brush border (Fig. 1D, arrows), and nuclei with the characteristic "fried egg" appearance of intestinal nuclei (Fig. 1D, arrowhead). Thus, based on cellular morphology, nearly all cells in the embryos resembled the intestinal cells of normal embryos. Wild-type embryos (not shown) and embryos carrying control transgenes expressing either the DAD-1 protein (a subunit of oligosaccharyl transferase) (Sugimoto et al. 1995; Kelleher et al. 1997) or a nonendodermal GATA factor (see below) under control of the heat shock promoters never showed apparently extra
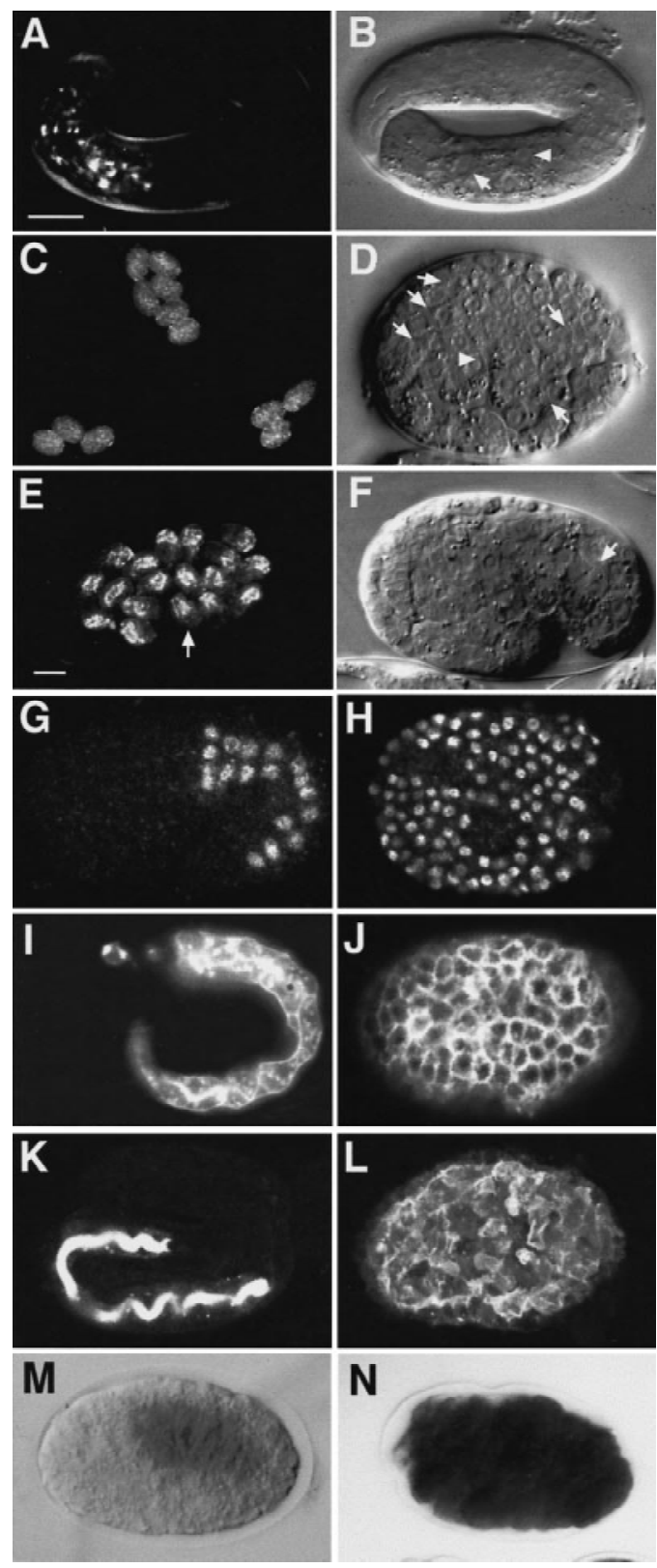

intestinal cells following the same heat-shock regime (not shown).

Ectopic end-1 expression was also found to promote widespread expression of biochemical markers characteristic of endoderm differentiation. ELT-2, a GATA factor that is required for proper formation of a functional gut and that is normally expressed in the early E lineage shortly after end-1 is activated (Fukushige et al. 1998), was present throughout embryos expressing end-1 ectopically (Fig. 1, cf. G and H). Similarly, two later-appearing antigens specific for the differentiated intestine, the first present throughout gut cells and recognized by antibody 1CB4 (Okamoto et al. 1985) and the second localized to the intestinal brush border and recognized by antibody MH33 (Waterston et al. 1988), were both detected ubiquitously in hs-end-1 embryos (Fig. 1, cf. I with 


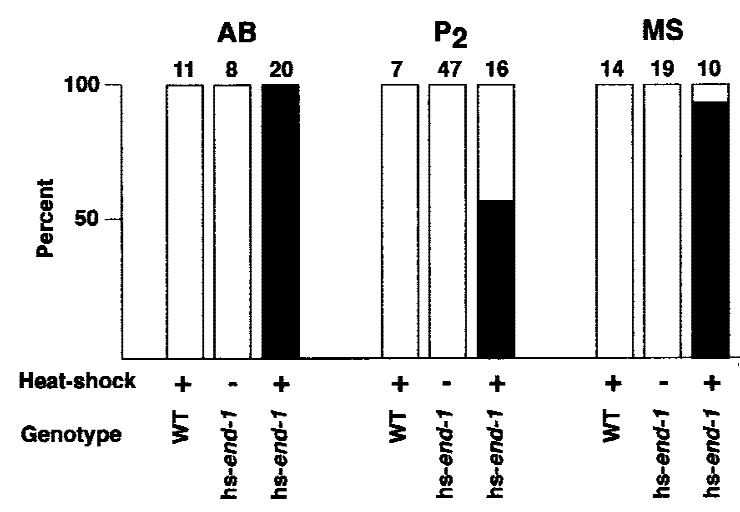

Figure 2. Ectopic production of gut from isolated early blastomeres. Differentiated gut was scored on the basis of cellular morphology and the presence of birefringent gut granules in developed partial embryos. The blastomere isolated and numbers of embryos analyzed are indicated above each set of bars. Embryos carrying hs-Ce-dad-1 were used as wild-type (WT) controls. (Open bars) Without gut; (solid bars) with gut.

J and $\mathrm{K}$ with L, respectively). The activity of the gutspecific GES-1 esterase (Edgar and McGhee 1986) was also detected throughout heat-shocked embryos (Fig. 1, cf. $\mathrm{M}$ and N). As these endoderm-specific molecules perform distinct biochemical functions, are localized to different subcellular compartments within endodermal cells, and are expressed at different times in embryogenesis, these results suggest that possibly most aspects of the endoderm program are activated by end-1 expression. Although the heat shock promoter probably results in levels of END-1 that are well above normal, possibly masking the role of other factors that regulate endoderm development, these findings show that ectopic END-1, at least at high levels, is sufficient to direct widespread endoderm differentiation.

\section{All early blastomeres can be transformed to endoderm precursors}

To assess whether all early blastomeres are capable of producing endoderm when END-1 is expressed ectopically, all normally nonendoderm-producing early blastomeres, that is, $\mathrm{AB}, \mathrm{P}_{2}$, and $\mathrm{MS}$, were each isolated from hs-end-1 embryos by laser-ablating all other cells; the resultant partial embryos were then assayed for endoderm production following heat shock (Fig. 2). Whereas blastomeres isolated from control embryos never expressed endodermal markers, all blastomeres isolated from heat-shocked hs-end-1 embryos frequently or always gave rise to endodermal markers (i.e., gut granules and the gut-specific antigen $\mathrm{MH} 33$ ), suggesting that each is capable of differentiating gut in response to end-1 expression (Fig. 2).

We asked whether nonendodermal cells can adopt an E cell lineage pattern by following cell lineages (not shown). In heat-shocked hs-end-1 embryos, the cellcycle timings in the early $\mathrm{AB}, \mathrm{MS}$, and $\mathrm{C}$ lineages resembled the timings in the normal $\mathrm{E}$ lineage, and programmed cell deaths, which always occur in these non-E somatic lineages (Sulston et al. 1983), were never observed in these embryos; both observations are consistent with a lineage transformation. Moreover, the numbers of nuclei present in these embryos were greatly diminished $(<200$ nuclei) compared with that in wild type ( 560 nuclei). This is consistent with an E-like transformation of other lineages, as the E lineage gives rise to far fewer cells than do any other somatic precursors. However, the reduced number of nuclei could also be a consequence of overexpressing GATA factors, because ectopic expression of two other GATA factors (Fukushige et al. 1998; see also Fig. 3) resulted in differentiated embryos with reduced numbers of nuclei (not shown). Overexpression of end-1 in heat-shocked embryos was also found to eliminate the asymmetries of the normal E lineage, which limited the value of cell division patterns as a criterion for cell fate transformation in these experiments.

To investigate further whether ectopic endoderm production might reflect transformations in early blastomere fates, we examined whether ectopic END-1 not
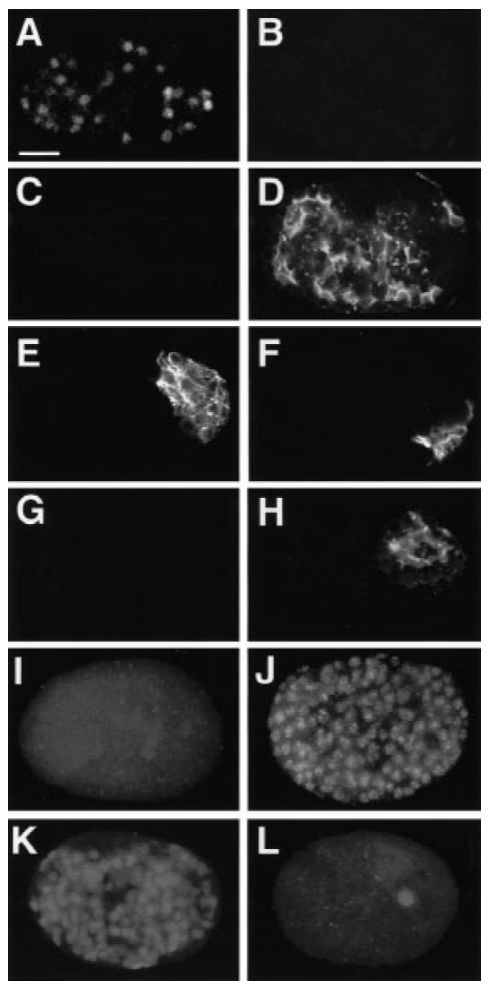

Figure 3. end-1 expression represses production of nonendodermal differentiation markers. $(A-H)$ Developed, partial embryos derived from isolated $\mathrm{AB}(A-D)$ and $\mathrm{P}_{2}(E-H)$ blastomeres. Differentiated partial embryos derived from each isolated blastomere without heat shock $(A, C, E, G)$ and after heat shock $(B, D, F, H)$ were stained for LIN-26 $(A, B)$, body-wall muscle $(E, F)$, and MH33 $(C, D, G, H)$. LIN-26 is a zinc-finger transcription factor normally expressed in all epidermal and neuron-associated support cells (Labouesse et al. 1996). (I-L) Whole embryos showing reciprocal effects of ectopic GATA factor expression. Representative heat-shocked hs-end-1 $(I, J)$ and hs-elt-1 $(K, L)$ embryos stained for LIN-26 $(I, K)$ and ELT-2 $(J, L)$ are shown. 
only activates expression of endodermal markers but also suppresses expression of markers characteristic of nonendodermal lineages. We found that expression of nonendodermal lineage markers from isolated blastomeres, including the LIN-26 ectodermal marker (Labouesse et al. 1996) from AB (Fig. 3A-D), body muscle from $\mathrm{P}_{2}$ (Fig. 3E-H), and pharynx muscle from MS (not shown), was attenuated in a heat shock-dependent manner in hs-end-1 embryos, concomitant with ectopic production of intestine. Thus, activation of endoderm development by END-1 apparently results in coincident repression of genetic programs that promote nonendodermal cell types.

We examined the specificity of this developmental reprogramming by asking whether a GATA factor that is not involved in endoderm specification causes a similar effect. The largely complete genomic sequence of C. elegans (C. elegans sequencing consortium, pers. comm.) predicts a minimum of 10 GATA factors in this organism (M. Maduro and J. Rothman, unpubl.). Although the functions of most of these factors are as yet unknown, at least three [END-1 (Zhu et al. 1997), END-3 (M. Maduro, E. Newman-Smith and J. Rothman, unpubl.), and ELT-2 (Fukushige et al. 1998)] appear to be endoderm-specific. Another GATA factor, ELT-1, is required for the development of an ectodermal cell type, the epidermis (Page et al. 1997). We compared the effects of ectopically expressing END-1 and ELT-1 on expression of the endodermspecific ELT-2 and ectoderm-specific LIN-26 proteins. As shown in Figure 3, I and J, whereas ectopic END-1 leads to widespread expression of ELT-2, most 163\%; $n=106)$ of the heat-shocked hs-end-1 embryos with 200 nuclei showed no detectable LIN-26, compared with the 11 to 39 LIN-26-expressing nuclei seen in wildtype embryos at similar stages (Labouesse et al. 1996). In contrast, expression of elt-1 from a heat shock promoter resulted in an effect that, albeit less dramatic, was reciprocal to that of end-1: $27 \%(7 / 26)$ of such embryos showed no detectable ELT-2 expression (e.g., Fig. 3L); $77 \%(20 / 26)$ showed no staining with the gut-specific MH33 antibody (not shown). However, all showed widespread, and apparently enhanced, expression of the LIN26 ectodermal marker (Fig. 3K). Moreover, ectopic expression of three other GATA factors that are not expressed in the endoderm repress gut differentiation (K. Koh, M. Maduro, and J. Rothman, unpubl.), whereas expression of the endoderm-specific ELT-2 and END-3 GATA factors also appear to promote widespread gut differentiation (Fukushige et al. 1998; M. Maduro and J. Rothman, unpubl.). These results suggest that the ability to reprogram early blastomeres into endoderm progenitors is limited to endoderm-specific GATA factors.

\section{Competency for reprogramming to endoderm occurs during a critical period in development}

We investigated whether the period of competency for reprogramming of cell fates by end-1 extends beyond the time that embryonic founder cells are born by performing heat shocks at various times in embryogenesis. As shown in Figure 4, embryos can produce ectopic intestine during an extended period. Embryos respond as early as the 12-cell stage, which probably reflects the earliest stage at which the heat shock promoter can be induced. In embryos heat-shocked after 200 cells, although expression from the heat shock promoter is still robust (Stringham et al. 1992), morphological characteristics (Fig. 1E, F) and molecular markers (not shown) of normal differentiation from all germ layers were seen, suggesting that most cells had become committed to their normal differentiated fates and were not capable of being reprogrammed by end-1. The optimal period for response ( 20-50 cells) coincides with the time at which end-1 is normally expressed in wild-type embryos (Zhu et al. 1997). Nonetheless, end-1 can cause a transformation in cell fate well beyond the stage at which the founder cell lineages are established and differentiation potential is normally restricted to particular blastomeres (Laufer et al. 1980; Cowan and McIntosh 1985; Edgar and McGhee 1986; Schnabel and Priess 1997; Bowerman 1998).

The requirement for maternal SKN-1 and the Wnt pathway in endoderm development can be bypassed

At least two maternal pathways, directed by nine known maternally active genes, are essential to specify endoderm in C. elegans. One pathway involves the maternal SKN-1 transcription factor (Bowerman et al. 1992; Blackwell et al. 1994), which is present in E and its progenitors. SKN-1 binds to end-1 regulatory sequences through SKN-1 consensus binding sites (Blackwell et al. 1994) that are apparently required for end-1 activation in the $\mathrm{E}$

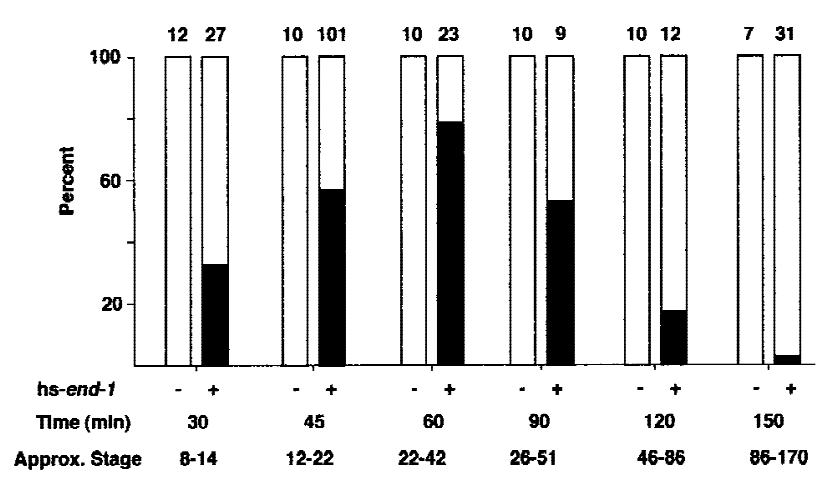

Figure 4. Time-course of competence for ectopic endoderm production. The presence of ectopic gut was scored in a control strain [hs-Ce-dad-1; (-)] or in hs-end-1 $(+)$ in embryos that had been heat-shocked at various times in development. The time between isolation of two- to four-cell embryos and the beginning of the heat shock is indicated. (Approx. Stage) Estimated developmental stage in numbers of cells at the time of the heat shock (Sulston et al. 1983). The proportions of embryos (solid bars) with widespread gut cells and those (open bars) containing gut restricted to one region of the embryo are given as percentages. Some heat-shocked hs-end-1 embryos appeared to contain extra gut cells but were included with those containing a normal gut because the gut granules were not distributed over the entire embryo; thus, the data shown somewhat underestimate the extent of ectopic gut formation. The number of embryos examined is indicated above each bar. 
lineage (J. Zhu, E. Witze, and J. Rothman, unpubl.). Whereas only $\sim 20 \%$ of embryos lacking maternal SKN-1 protein make any endoderm (Bowerman et al. 1992), we found that $100 \%(38 / 38)$ of skn-1 mutant embryos expressing end-1 from the heat shock promoter made large amounts of endoderm; these embryos were indistinguishable from the $s k n-1(+)$ embryos expressing the construct (not shown). Thus, expression of end-1 eliminates the requirement for maternal SKN-1 in endoderm specification.

Endoderm production also requires a maternal Wnt signaling pathway that polarizes EMS, the parent of E (Rocheleau et al. 1997; Thorpe et al. 1997). We tested whether expression of end-1 can bypass the requirement for this Wnt pathway in endoderm specification by depleting embryos of maternal wrm-1 product (Rocheleau et al. 1997), a C. elegans homolog of the Wnt signal transduction component $\beta$-catenin/Armadillo (Peifer and Wieschaus 1990), with the technique of RNA interference (RNAi; Fire et al. 1998). wrm-1(RNAi) embryos almost never (1/135 embryos) produce differentiated intestine (Fg. 5A,C,E). In contrast, nearly all (54/56) such wrm-1-depleted embryos expressing end-1 from the heat-shock construct made differentiated intestine (Fig. $5 \mathrm{~B}, \mathrm{D}, \mathrm{F})$. Thus, endoderm development can be made independent of the maternal Wnt pathway by forcing expression of end-1.

Our findings suggest that the maternal Wnt pathway and maternal SKN-1 might well perform no other essential function in endoderm formation beyond activating expression of end-1 (and perhaps other proximal zygotic regulators) specifically within the E lineage. These zygotic regulators are in turn sufficient to activate the program for endoderm development.
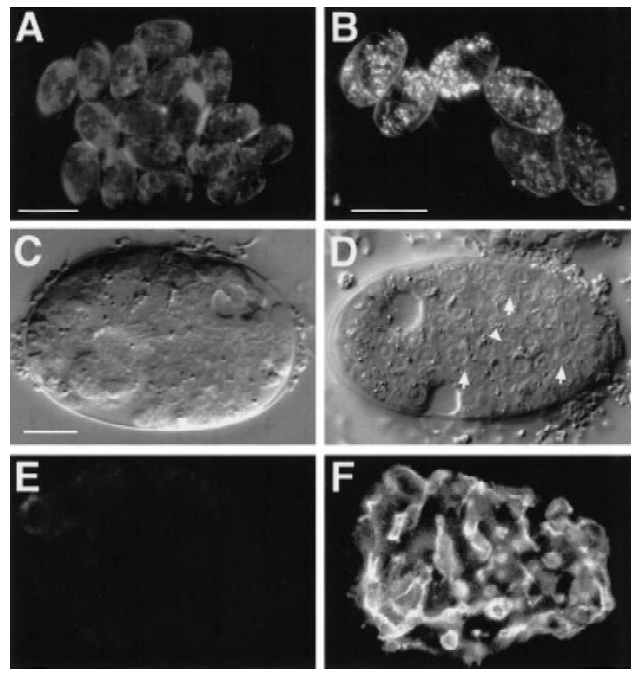

Figure 5. Forced end-1 expression overrides the requirement for wrm-1 activity. Essentially all nonheat-shocked hs-end-1; wrm-1(RNAi) embryos lacked birefringent gut granules $(A)$ and gut-like cells $(C)$ and failed to express the MH33 antigen $(E)$. When these embryos were given a heat shock, nearly all showed widespread gut granules $(B)$, gut-like cells (arrowhead in $D$ ) and brush-border structures (arrows in $D$ ), and MH33 expression $(F)$.

\section{Commitment to germ layer differentiation and exclusivity of cell fate}

Many studies of C. elegans embryogenesis have demonstrated how the potential to undergo particular pathways of differentiation becomes restricted to the founder cell lineages during early cleavages (for review, see Schnabel and Priess 1997; Bowerman 1998). Our results indicate that this restriction does not reflect an irreversible commitment: By forcing expression of a zygotic endoderm regulator, founder cell lineages that normally do not inherit the capacity to make endoderm can be redirected into an endoderm lineage well after they are established. At what stage lineages become irreversibly committed to a particular developmental pathway remains an open question. Once a cell has adopted a particular differentiated fate, however, there appear to be mechanisms that exclude all other fates; in particular, when cell identities are altered by experimental manipulation (e.g., Cowan and McIntosh 1985; Boshart et al. 1993), cells generally express genes characteristic of only a single differentiated cell type. This mutual exclusivity of cell fates presumably involves a network of gene activity that not only activates genes appropriate to that cell type but represses all others, thereby ensuring an either-or decision. We observed such behavior reciprocally with the endodermal END-1 and the ectodermal ELT-1 GATA factors. It should be possible to reveal the mechanism of this mutual exclusivity by dissecting the entire network of regulators that collaborate to direct endoderm development.

\section{Materials and methods}

Constructs and transgenic animals Primers JZ27 (5'-CCCTAGCTAGCATGTATCAATCGTCTTCTTCATCTCC3-') and JZ30 (5'-GGGGTACCGGAGCATACACATAAAAAGATAAG-3') were used to amplify an end-1 cDNA fragment from pJZ9 (Zhu et al. 1997). Plasmids pJZ32a and pJZ32b were constructed by placing two independently PCR-amplified end-1 cDNA fragments ( $a$ and b) downstream of the hsp16-2 promoter as follows: Amplified PCR products were cut with NheI and KpnI and subcloned into NheI-KpnI sites of pPD49.78 vector provided by A. Fire (Carnegie Institute, Baltimore, MD). Plasmids pJZ33a and pJZ33b were constructed in a similar way except that the vector pPD49.83 was used. The hs-elt-1 construct is described in Fukushige et al. (1998). Because hsp16-2 and hsp16-41 are expressed optimally in different tissues (Stringham et al. 1992), transgenic animals carrying both the hsp16-2::end-1 and hsp16-41::end-1 constructs were generated to ensure broad expression of the gene. A mix of pJZ32a,b and pJZ33a,b (each plasmid at $\sim 2.5 \mu \mathrm{g} / \mathrm{ml}$ ) was coinjected with a marker plasmid pRF4 [rol-6(su1006) (Mello et al. 1991)] at $100 \mu \mathrm{g} / \mathrm{ml}$. To integrate the resulting transgenic array $(w E x 180)$, the transgenic animals were $\gamma$-irradiated followed by triple backcrossing to the wild-type N2 strain. Strains homozygous for each integrant were used. The genotypes of strains used in this study are JR662 (N2; wIs47), JR663 (N2; wIs48), JR665 (N2; wIs50), and JJ197 [unc-5(e53) skn-1(zu67/dpy-13(e184sd) unc-8(n491sd)]. JR662 and JR663 were crossed with JJ197 and gravid Unc Rol adults were selected for the heat shock experiments.

\section{Analysis of gut differentiation}

Embryos at the two- to four-cell stage were dissected from Rol hermaphrodites of the appropriate genotype and mounted on an agar pad as described (Sulston et al. 1983). Embryos were allowed to develop at $23 \pm 1^{\circ} \mathrm{C}$ for $1 \mathrm{hr}$ (Fig. 2) or the indicated times (Figs. 1 and 4), heatshocked at $33-34^{\circ} \mathrm{C}$ for $30 \mathrm{~min}$, and incubated at $20^{\circ} \mathrm{C}$ overnight. The presence of gut granules was assayed by polarized light microscopy. Immunofluorescence was performed as described elsewhere (Zhu et al. 1997) and images collected with a Bio-Rad 2400 confocal microscope. 
Laser cell ablation

A VSL-337 nitrogen laser (Laser Science) attached to a laser ablation unit (Photonics Instruments, Arlington Heights, IL) was used for ablations. Ablations were performed at $22-23^{\circ} \mathrm{C}$ followed by incubation for the appropriate time prior to heat shock. The fraction of partial embryos containing birefringent gut granules was scored after they were allowed to develop overnight at $20^{\circ} \mathrm{C}$. The $\mathrm{AB}$ blastomere was isolated by ablating $\mathrm{P}_{2}$ and EMS. The MS blastomere was isolated by first ablating $\mathrm{ABa}, \mathrm{ABp}$, and $\mathrm{P}_{2}$, followed by ablation of $\mathrm{E}$ after EMS divided. The $\mathrm{P}_{2}$ blastomere was isolated by ablating $\mathrm{ABa}, \mathrm{ABp}$, and EMS. Although $\mathrm{P}_{2}$ is a precursor of both germ-line and somatic tissue, the exclusive germ-line progenitor $\left(\mathrm{P}_{4}\right)$ was not tested per se, because zygotic transcription is generally repressed in the germ-line lineage (Seydoux et al. 1996).

RNAi

PCR primers specific for a wrm-1 sequence (5'-AATACGACTCACTATAGGGCTCGAGCGGCCGCCCGGGCAGGT-3') were used to amplify a wrm-1 cDNA from an embryonic cDNA pool made according to the Marathon RACE-PCR kit (Clontech Marathon Kit). An 780-bp PCR fragment was subcloned into vector pCR2.1 (Stratagene), creating pIZ50B. Antisense wrm-1 RNA was made from linearized pJZ50B and injected into young JR663 adults at $\sim 0.5 \mu \mathrm{g} / \mu \mathrm{l}$. Injected adults were incubated overnight at $20^{\circ} \mathrm{C}$, collected on a fresh plate, and embryos laid between 18 and $24 \mathrm{hr}$ were collected onto an agar pad and allowed to develop overnight without heat shock. Embryos laid after $24 \mathrm{hr}$ were collected every $15 \mathrm{~min}$, mounted onto an agar pad, and heat-shocked immediately for $30 \mathrm{~min}$ at $33-34^{\circ} \mathrm{C}$, followed by overnight incubation at $20^{\circ} \mathrm{C}$. Gut granules, morphology, and $\mathrm{MH} 33$ reactivity were scored on developed embryos.

\section{Acknowledgments}

Monoclonal antibodies NE8/4C6, 3NB12, and 1CB4 were obtained from the MRC-Cambridge collection, MH33 was a gift from R. Waterston, and anti-LIN-26 antibody was a gift from M. Labouesse. Some of the strains used in this work were provided by the Caenorhabditis Genetics Center, which is funded by the National Institutes of Health (NIH) National Center for Research Resources. This work was supported by grants from the NIH, the National Science Foundation, and the March of Dimes to J.H.R. and from the Medical Research Council of Canada to J.D.M and the Alberta Heritage Foundation for Medical Research to T.F. and J.D.M.

The publication costs of this article were defrayed in part by payment of page charges. This article must therefore be hereby marked 'advertisement' in accordance with 18 USC section 1734 solely to indicate this fact.

\section{References}

Blackwell, T.K., B. Bowerman, J.R. Priess, and H. Weintraub. 1994. Formation of a monomeric DNA binding domain by Skn-1 bZIP and homeodomain elements. Science 266: 621-628.

Boshart, M., D. Nitsch, and G. Schutz. 1993. Extinction of gene expression in somatic cell hybrids-a reflection of important regulatory mechanisms? Trends Genet. 9: 240-245.

Bossinger, O. and E. Schierenberg. 1992. Transfer and tissue-specific accumulation of cytoplasmic components in embryos of Caenorhabditis elegans and Rhabditis dolichura: In vivo analysis with a low-cost signal enhancement device. Development 114: 317-330.

Bowerman, B. 1998. Maternal control of pattern formation in early Caenorhabditis elegans embryos. Curr. Top. Dev. Biol. 39: 73-117.

Bowerman, B., B.A. Eaton, and J.R. Priess. 1992. skn-1, a maternally expressed gene required to specify the fate of ventral blastomeres in the early C. elegans embryo. Cell 68: 1061-1075.

Bowerman, B., B.W. Draper, C.C. Mello, and J.R. Preiss. 1993. The maternal gene $s k n-1$ encodes a protein that is distributed unequally in early C. elegans embryos. Cell 74: 443-452.

Cowan, A.E. and J.R. McIntosh. 1985. Mapping the distribution of differentiation potential for intestine, muscle, and hypodermis during early development in Caenorhabditis elegans. Cell 41: 923-932.

Edgar, L.G. and J.D. McGhee. 1986. Embryonic expression of a gut-specific esterase in Caenorhabditis elegans. Dev. Biol. 114: 109-118.
Fire, A., S. Xu, M.K. Montgomery, S.A. Kostas, S.E. Driver, and C.C Mello. 1998. Potent and specific genetic interference by doublestranded RNA in Caenorhabditis elegans. Nature 391: 806-811.

Fukushige, T., M.G. Hawkins, and J.D. McGhee. 1998. The GATA-factor ELT-2 is required for development of the C. elegans gut. Dev. Biol. 198: $286-302$.

Kelleher, D.J. and R. Gilmore. 1997. DAD1, the defender against apoptotic cell death, is a subunit of the mammalian oligosaccharyltransferase. Proc. Nat1. Acad. Sci. 94: 4994-4999.

Labouesse, M., E. Hartwieg, and H.R. Horvitz. 1996. The Caenorhabditis elegans LIN-26 protein is required to specify and/or maintain all non-neuronal ectodermal cell fates. Development 122: 2579-2588.

Laufer, J.S., P. Bazzicalupo, and W.B. Wood. 1980. Segregation of developmental potential in early embryos of Caenorhabditis elegans. Cell 19: $569-577$.

Laverriere, A.C., C. MacNeill, C. Mueller, R.E. Poelmann, J.B.E. Burch, and T. Evans. 1994. GATA-4/5/6, a subfamily of three transcription factors transcribed in developing heart and gut. I. Biol. Chem. 269: $23177-23184$.

Mello, C.C., J.M. Kramer, D. Stinchcomb, and V. Ambros. 1991. Efficient gene transfer in C. elegans: Extrachromosomal maintenance and integration of transforming sequences. EMBO J. 10: 3959-3970.

Okamoto, H. and J.N. Thomson. 1985. Monoclonal antibodies which distinguish certain classes of neuronal and supporting cells in the nervous tissue of the nematode Caenorhabditis elegans. J. Neurosci. 5:643-653

Page, B.D., W. Zhang, K. Steward, T. Blumenthal, and J.R. Priess. 1997. ELT-1, a GATA-like transcription factor, is required for epidermal cell fates in Caenorhabditis elegans embryos. Genes \& Dev. 11: $1651-1661$.

Peifer, M. and E. Wieschaus. 1990. The segment polarity gene armadillo encodes a functionally modular protein that is the Drosophila homolog of human plakoglobin. Cell 63: 1167-1176.

Rehorn, K.P., H. Thelen, A.M. Michelson, and R. Reuter. 1996. A molecular aspect of hematopoiesis and endoderm development common to vertebrates and Drosophila. Development 122: 4023-4031.

Reuter, R. 1994. The gene serpent has homeotic properties and specifies endoderm versus ectoderm within the Drosophila gut. Development 120: $1123-1135$

Rocheleau, C.E., W.D. Downs, R. Lin, C. Wittmann, Y. Beri, Y.H. Cha, M. Ali, J.R. Priess, and C.C. Mello. 1997. Wnt signaling and an APCrelated gene specify endoderm in early C. elegans embryos. Cell 90: $707-716$.

Schnabel, R. and J.R. Priess. 1997. Specification of cell fates in the early embryo. In C. elegans II (ed. D.L. Riddle), pp. 361-382. Cold Spring Harbor Laboratory Press, Cold Spring Harbor, NY.

Seydoux, G., C.C. Mello, J. Pettitt, W.B. Wood, J.R. Priess, and A. Fire. 1996. Repression of gene expression in the embryonic germ lineage of C. elegans. Nature 382: 713-716.

Siddiqui, S.S. and P. Babu. 1980. Kynurenine hydroxylase mutants of the nematode Caenorhabditis elegans. Mol. \& Gen. Genet. 179: 21-24.

Soudais, C. 1995. Targeted mutagenesis of the transcription factor GATA-4 gene in mouse embryonic stem cells disrupts visceral endoderm differentiation in vitro. Development 121: 3877-3888.

Stringham, E.G., D.K. Dixon, D. Jones, and E.P. Candido. 1992. Temporal and spatial expression patterns of the small heat shock (hsp16) genes in transgenic Caenorhabditis elegans. Mol. Biol. Cell. 3: 221-233.

Sugimoto, A., R.R. Hozak, T. Nakashima, T. Nishimoto, and J.H. Rothman. 1995. dad-1, an endogenous programmed cell death suppressor in Caenorhabditis elegans and vertebrates. EMBO J. 14: 4434-4441.

Sulston, J.E., E. Schierenberg, J.G. White, and J.N. Thomson. 1983. The embryonic cell lineage of the nematode Caenorhabditis elegans. Dev. Biol. 100: 64-119.

Thorpe, C.J., A. Schlesinger, J.C. Carter, and B. Bowerman. 1997. Wnt signaling polarizes an early C. elegans blastomere to distinguish endoderm from mesoderm. Cell 90: 695-705.

Waterston, R.H. 1988. Muscle. In The Nematode Caenorhabditis elegans (ed. W.B. Wood). Cold Spring Harbor Laboratory, Cold Spring Harbor, NY.

Zhu, J., R.J. Hill, P.J. Heid, M. Fukuyama, A. Sugimoto, J.R. Priess, and J.H. Rothman. 1997. end-1 encodes an apparent GATA factor that specifies the endoderm precursor in Caenorhabditis elegans embryos. Genes \& Dev. 11: 2883-2896. 


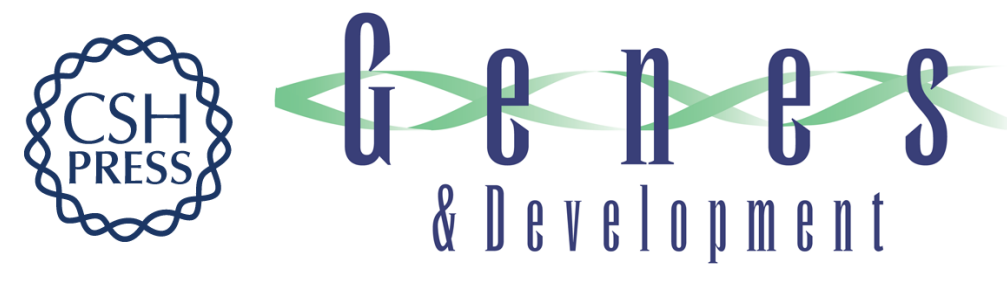

\section{Reprogramming of early embryonic blastomeres into endodermal progenitors by a Caenorhabditis elegans GATA factor}

Jiangwen Zhu, Tetsunari Fukushige, James D. McGhee, et al.

Genes Dev. 1998, 12:

Access the most recent version at doi:10.1101/gad.12.24.3809

References

This article cites 29 articles, 12 of which can be accessed free at:

http://genesdev.cshlp.org/content/12/24/3809.full.html\#ref-list-1

License

Email Alerting

Receive free email alerts when new articles cite this article - sign up in the box at the top Service right corner of the article or click here.

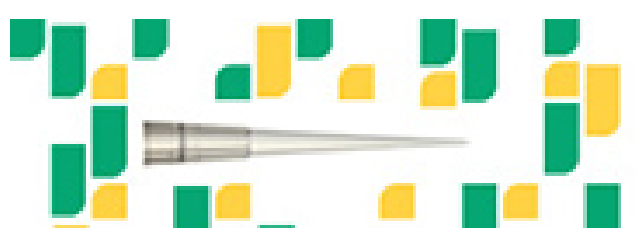

Focused on your science. 